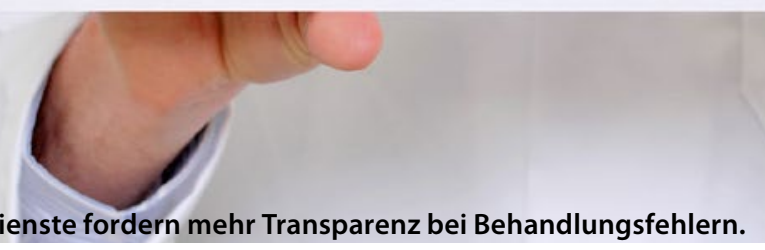

\title{
Jahresstatistik
}

\section{So viele Behandlungsfehler gab es 2015}

\section{Die Medizinischen Dienste haben eine Behandlungsfehlerstatistik vor- gelegt. Die meisten Beschwerden gab es nach Operationen.}

V ertreter der Medizinischen Dienste der Krankenkassen (MDK) haben eine Meldepflicht für Behandlungsfehler gefordert. „Die tatsächliche Anzahl der Behandlungsfehler ist nicht bekannt", sagte Dr. Stefan Gronemeyer, Leitender Arzt beim Dienst des GKV-Spitzenverbandes bei der Vorstellung der Behandlungsfehlerstatistik für 2015 im Mai in Berlin. Mit einer systematischen Erfassung ließen sich die Statistiken der Medizinischen Dienste, der Schlichtungsstellen der Ärzteschaft, der Haftpflichtversicherer, der Gerichte und Rechtsanwälte zusammenfassen.

Der größte Datensatz liegt den Medizinischen Diensten vor. Ihre Gutachter wurden 2015 in rund 14.800 Fällen gefordert, von Patienten erhobene Vorwürfe zu prüfen. Das waren $200 \mathrm{mehr}$ als im Jahr zuvor. 4.026 Mal bestätigten die Gutachter den Verdacht der Patienten, auch diese Zahl stieg im Vergleich zu 2014, als noch 3.796 Fehler bestätigt wurden. 3.156 Mal haben Fehler im vergangenen Jahr direkt einen Schaden bei Patienten ausgelöst, zeigt die MDK-Statistik. 125 Menschen starben infolge von Behandlungsfehlern.

„Die Zahlen sind nur die Spitze des Eisbergs", sagte Gronemeyer. Dieser Einschätzung schloss sich in einer ersten Reaktion die Grünen-Politikerin
Maria Klein-Schmeink an. Es fehle an Transparenz. Die Politikerin erneuerte ihre Forderung nach der Einrichtung eines Härtefallfonds im Patientenrechtegesetz.

Das Gros der Beschwerden, in Zahlen 9.899, sei nach Operationen in Krankenhäusern eingegangen, berichteten die MDK-Vertreter. Fehler seien dort für Patienten leichter erkennbar als zum Beispiel bei Diagnosefehlern in Praxen niedergelassener Ärzte. Im ambulanten Sektor wurde 4.905 Vorwürfen nachgegangen.

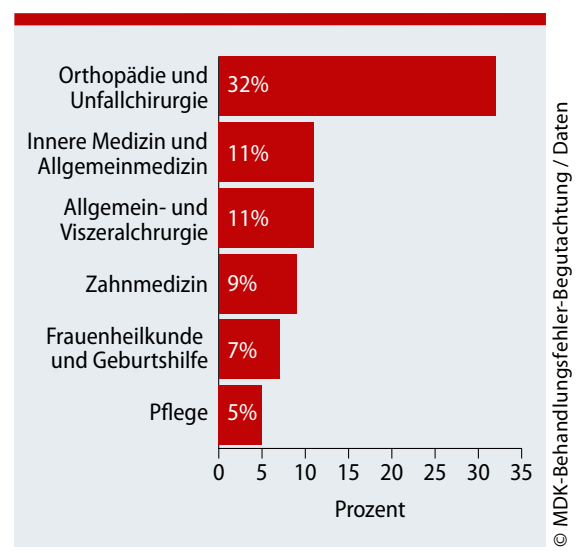

Verteilung der Vorwürfe auf Fachgebiete. Die fehlenden 25 Prozent verteilen sich auf insgesamt 33 weitere Fachgebiete.
Die Fehlerstatistik der Bundesärztekammer (BÄK) war im März zu vergleichbaren Ergebnissen gekommen. Hier war die Zahl der 2015 festgestellten Behandlungsfehler (2.132) im Vergleich zum Vorjahr (2.252) zwar leicht gesunken, mit insgesamt 11.822 Anträgen bei den Schlichtungsstellen für Arzthaftpflichtfragen waren aber deutlich mehr Verdachtsmomente geäußert worden. Als Grund wurde das Patientenrechtegesetz angegeben, das seit drei Jahren in Kraft ist.

In beiden Statistiken sind vor allem Unfallchirurgen und Orthopäden betroffen: In der aktuellen Statistik der MDK-Gemeinschaft bezogen sich $32 \%$ aller Vorwürfe auf Orthopädie und Unfallchirurgie, $11 \%$ auf die Innere Medizin und Allgemeinmedizin und weitere $11 \%$ auf die Allgemeinchirurgie.

\section{Pflege: jeder zweite Verdacht wahr} $5 \%$ der Vorwürfe wurden in der Pflege geäußert. Hier fällt auf, dass in mehr als 52 \% der Fälle tatsächlich ein Fehler festgestellt wurde: 768 eingegangenen Vorwürfen lagen 403 Behandlungsfehler zugrunde.

Im Verhältnis zur Gesamtzahl der medizinischen und pflegerischen Behandlungen liegt die Zahl der Behandlungsfehler jedoch niedrig. Der stationäre Sektor verzeichnet rund 19 Millionen Behandlungen, die niedergelassenen Ärzte 550 Millionen Behandlungen im Jahr. Anno Fricke, Jana Kötter 